\title{
Establishment of a rat autogenous arteriovenous fistula model following 5/6 nephrectomy
}

\author{
CHENFEI ZHENG, YING ZHOU, CHAOXING HUANG, ZHOUCHANG ZHANG, YI LIU and YULAN XU \\ Department of Nephrology, The First Affiliated Hospital of Wenzhou Medical University, \\ Wenzhou, Zhejiang 325000, P.R. China
}

Received August 2, 2014; Accepted March 5, 2015

DOI: 10.3892/etm.2015.2451

\begin{abstract}
The aim of this study was to establish a stable rat model of autogenous arteriovenous fistula (AVF) with chronic renal function insufficiency. Forty Sprague-Dawley rats were randomly divided into an experimental group $(n=20)$ and sham surgery group $(n=20)$ and a 5/6 nephrectomy model was established in the rats. One week later, in the experimental group, the ipsilateral caroid artery was inserted into the external jugular vein by a cuff technique to establish a carotid arteriovenous fistula; in the sham group, the external jugular vein and carotid artery were dissociated. At 7 and 28 days following the establishment of the AVF, the renal functions of the two groups were measured. Hematoxylin and eosin staining and double collagen and elastin staining were conducted to evaluate the area of intimal hyperplasia in the external jugular vein, and the expression of $\alpha$-smooth muscle actin in the vein was investigated by immunohistochemistry. The creatinine and urea nitrogen levels in the experimental group at each time-point were significantly higher than those in the sham surgery group $(\mathrm{P}<0.05)$. The intimal hyperplasia of the external jugular vein of the experimental group was increased significantly compared with that in the sham group at each time-point $(\mathrm{P}<0.05)$. The model, which is easy to establish and simple to master, provides a new and feasible experimental method for the study of intimal hyperplasia associated with autogenous AVF in chronic renal insufficiency, and is worthy of wider use.
\end{abstract}

\section{Introduction}

Autogenous arteriovenous fistula (AVF) is considered the best type of vascular access for maintenance hemodialysis (1). In patients with chronic renal insufficiency, immature AVFs are

Correspondence to: Dr Chenfei Zheng, Department of Nephrology, The First Affiliated Hospital of Wenzhou Medical University, 2 Fuxue Lane, Wenzhou, Zhejiang 325000, P.R. China

E-mail: chenfeizhengen@163.com

Key words: animal model, venous fistula arteriovenous fistula, intimal hyperplasia, 5/6 nephrectomy observed when significant anatomical features cause a failure to mature (2), and intimal hyperplasia seriously affects the long-term efficacy (3). Routine preoperative upper extremity mapping with ultrasound increases not only the AVF construction rate, but also the likelihood of maturation (4). To predict the function of AVFs, intraoperative ultrasound transit-time flow measurements gained at surgery and postoperative follow-up with duplex ultrasonography have been found to be helpful (5). Animal models of fistula can be used for observation of the changes in the local flow dynamics of arteries and pathological dynamic changes of venous arterialization, and to investigate intervention effects. Large and small animals have advantages and disadvantages in modeling. Fistula models using large animals such as dogs and pigs, which have vascular structures similar to those of humans and are simple to establish, provide a satisfactory simulation of local hemodynamics. It is also easy to perform external stent intervention and the monitoring of some hemodynamic data in such models. However, large animals have high feeding costs and a complex process of anesthesia, and it can be difficult to establish a mixed model of disease, such as with diabetes or chronic renal failure. Small animal models do not have these deficiencies (6-9), and the wide use of gene knockout rats in recent years is particularly important, as it provides an important platform for the study of the mechanisms of the occurrence and development of diseases. The establishment of an arteriovenous fistula model in animals with chronic renal failure by a cuff technique has been reported (10), but the experimental results are often influenced by the technology proficiency level of the practitioner. A rat model of arterial bypass grafting has suggested that the hemodynamic and morphological changes associated with a distal AVF may potentiate graft patency and function (11). In the present study, an AVF model was established using a cuff technique following $5 / 6$ nephrectomy.

\section{Materials and methods}

Experimental animals. A total of 40 specific pathogen-free healthy male Sprague-Dawley (SD) rats, 4 weeks old, weighing 150-160 g were purchased from Shanghai Silaike Experimental Animal Co. Ltd. (Shanghai, China). The rats were fed in the laminar laboratory of the Experimental Animal Center of Wenzhou Medical College (Wenzhou, China), with a feeding temperature of $25^{\circ} \mathrm{C}$, relative humidity 
of $70 \%$ and a 12/12 h light/dark cycle. The study was carried out in strict accordance with the recommendations in the Guide for the Care and Use of Laboratory Animals of National Research Council (12). The animal use protocol has been reviewed and approved by the Institutional Animal Care and Use Committee (IACUC) of Wenzhou Medical University.

Cuff. A $20 \mathrm{G}$ disposable venous indwelling needle catheter (B. Braun Melsungen AG, Melsungen, Germany), cut into 2.5-mm segments was used.

Establishment of the 5/6 nephrectomy rat model. Following a week of adaptive feeding, random digital expression methods were used to randomly divide the rats into an experimental group $(n=20)$ and sham surgery group $(n=20)$, and the modeling method was conducted according to the previously reported method $(13,14)$.

Establishment of the rat AVF model. A week after the establishment of the 5/6 nephrectomy rat model, the rats of the experimental group were anesthetized by the intraperitoneal injection of $300 \mathrm{mg} / \mathrm{kg} 10 \%$ chloral hydrate aqueous solution. The neck skin was shaved, and a $\sim 1.5 \mathrm{~cm}$ oblique incision was made in the neck slightly to the right of center. Following the freeing and resection of the right submandibular gland and right sternocleidomastoid muscle, the right external jugular vein injury was separated by a noninvasive technique. The branches were ligated with an 8-0 suture, an $\sim 8-\mathrm{mm}$ vein bridge length was dissociated, and the lumen was flushed with physiological saline (containing heparin sodium $5,000 \mathrm{U} / \mathrm{l})$. Then, $\sim 8 \mathrm{~mm}$ right common carotid artery was separated, and following ligation of the proximal end to block the blood flow with a noninvasive vascular clamp, the artery was cut in the middle point. The vessel wall at the broken end of the artery was passed through a disposable intravenous indwelling needle catheter; with gentle pulling, the artery was flipped outwards to sheathe the catheter vessel, and was then inserted into the reversed vein bridge. Ligation with 5-0 suture was twice performed, and the incision was sutured. In the control group, the same incision position was adopted, and following the freeing and resection of the right submandibular gland and right sternocleidomastoid muscle, the right external jugular vein and the right carotid artery were separated by a noninvasive technique, and the incision was sutured. The experimental animals were sent to the breeding center for recovery.

Specimen collection and treatment. All rats were fed with a standard pellet diet, with free access to food and water. On days 7 and 28 after the establishment of the AVF model, rats from the two groups were sacrificed (10 rats from each group at each time-point). Referring to the anastomotic stoma as the midpoint, $1 \mathrm{~cm}$ venous and arterial tissue was excised to be fixed with $4 \%$ paraformaldehyde overnight and embedded in paraffin prior to the preparation of 5- $\mu \mathrm{m}$ serial sections that were observed by an AX70 light microscope (Olympus Corp., Tokyo, Japan). The rats were sacrificed following the drawing of blood from the inferior vena cava; the blood was retained for analysis.
Determination of renal function. The dynamic changes of blood creatinine and urea nitrogen levels were monitored using an 7180 automatic biochemical analyzer (Hitachi High-Technologies Corp., Tokyo, Japan).

Histomorphology. The intimal tissues were subjected to hematoxylin and eosin staining, and collagen and elastin double staining with Victoria Blue B and Ponceau S staining solution (Fuzhou Maixin Biotechnology Development Co. Ltd., Fuzhou, China). Image Pro-Plus 6 software (Media Cybernetics, Inc., Rockville, MD, USA) was used for image analysis. The tunica intima and tunica media membrane thickness was respectively calculated, and the area of intimal hyperplasia was the area of the internal elastic lamina minus the area of the lumen.

Immunohistochemistry. The sections were routinely dewaxed and hydrated, and then subjected to $120^{\circ} \mathrm{C}$ antigen retrieval for $10 \mathrm{~min}$. Endogenous peroxidase activity was quenched with $3 \% \mathrm{H}_{2} \mathrm{O}_{2}$ for $10 \mathrm{~min}$, and then immune bovine serum was added for $10 \mathrm{~min}$ for blocking. The sections were then incubated with primary antibody (monoclonal mouse anti-rat $\alpha$-SMA antibody, 1:300, cat. no. 555018; Abcam, Cambridge, UK), at $4^{\circ} \mathrm{C}$ overnight. The sections were washed three times with phosphate-buffered saline (Sigma-Aldrich, St Louis, MO, USA), 5 min each time. After warming in a $37^{\circ} \mathrm{C}$ incubator for $20 \mathrm{~min}$, the secondary antibody (horseradish peroxidase-conjugated polyclonal goat anti-mouse IgG, cat. no. B-14029; Beijing Sequoia Jinqiao Biological Technology Co. Ltd., Beijing, China) was added and the sections were incubated at $37^{\circ} \mathrm{C}$ for $30 \mathrm{~min}$. The sections were then developed with 3,3'-diaminobenzidine, counterstained with hematoxylin, differentiated with $0.1 \%$ hydrochloric acid in alcohol, and flushed with water. The sections were then routinely dehydrated, mounted with transparent neutral gum and observed in Q550CW image acquisition system (Leica Science Lab, Berlin, Germany). In each vessel organization, three non-consecutive slices were observed and 10 high magnification (x200) fields of view were randomly selected in each section. The positive immunohistochemical staining signal of the selected view was subjected to image analysis, using Image Pro-Plus 6 image analysis software (MediaCybernetics, Inc., Rockville, MD, USA). The percentage of the positively stained area in relation to the total field area in each field, namely the $\alpha$-SMA relative positive area (\%) was calculated, and a mean value was then calculated.

Statistic analysis. Using SPSS software, version 16.0 (SPSS, Inc., Chicago, IL, USA) the measurement data were represented as mean \pm standard deviation. After testing for homogeneity of variance, single factor analysis of variance was performed for significance analysis. $\mathrm{P}<0.05$ was considered to indicate a statistically significant difference.

\section{Results}

Comparison of renal function. The creatinine and urea nitrogen levels of the experimental group on days 7 and 28 after modeling were significantly increased compared with 
Table I. Comparison of renal function in the rats after surgery.

\begin{tabular}{lcccc}
\hline & \multicolumn{2}{c}{ BUN $(\mathrm{mmol} / \mathrm{l})$} & \multicolumn{2}{c}{$\mathrm{SCr}(\mu \mathrm{mol} / \mathrm{l})$} \\
\cline { 2 - 5 } Time-point & $\begin{array}{c}\text { Experimental } \\
\text { group }\end{array}$ & $\begin{array}{c}\text { Sham surgery } \\
\text { group }\end{array}$ & $\begin{array}{c}\text { Experimental } \\
\text { group }\end{array}$ & $\begin{array}{c}\text { Sham surgery } \\
\text { group }\end{array}$ \\
\hline Day 7 & $13.37 \pm 1.07^{\mathrm{a}}$ & $6.32 \pm 1.12$ & $61.25 \pm 3.09^{\mathrm{a}}$ & $36.25 \pm 2.85$ \\
Day 28 & $15.12 \pm 1.42^{\mathrm{a}}$ & $7.36 \pm 1.85$ & $56.89 \pm 3.41^{\mathrm{a}}$ & $39.56 \pm 3.03$ \\
\hline
\end{tabular}

Values presented are the mean \pm standard deviation ( $\mathrm{n}=10$ per group). ${ }^{\mathrm{a}} \mathrm{P}<0.05$ compared with the sham surgery group at the same time-point. BUN, blood urea nitrogen; SCr, serum creatinine.

Table II. Vein pathological changes near the anastomotic stoma following surgery of each group of rats.

\begin{tabular}{|c|c|c|c|c|}
\hline \multirow[b]{2}{*}{ Variable } & \multicolumn{2}{|c|}{ Day 7} & \multicolumn{2}{|c|}{ Day 28} \\
\hline & $\begin{array}{l}\text { Experimental } \\
\text { group }\end{array}$ & $\begin{array}{c}\text { Sham surgery } \\
\text { group }\end{array}$ & $\begin{array}{c}\text { Experimental } \\
\text { group }\end{array}$ & $\begin{array}{c}\text { Sham surgery } \\
\text { group }\end{array}$ \\
\hline Tunica intima thickness $(\mu \mathrm{m})$ & $38.22 \pm 1.39^{\mathrm{a}}$ & $7.01 \pm 1.40$ & $69.50 \pm 5.34^{\mathrm{a}}$ & $8.34 \pm 1.22$ \\
\hline Tunica media thickness $(\mu \mathrm{m})$ & $50.51 \pm 2.65^{\mathrm{a}}$ & $21.82 \pm 2.25$ & $46.14 \pm 6.59^{\mathrm{a}}$ & $23.92 \pm 2.26$ \\
\hline Hyperplastic area $\left(\mu \mathrm{m}^{2}\right)$ & $19.20 \pm 1.30^{\mathrm{a}}$ & $2.39 \pm 1.56$ & $22.88 \pm 3.35^{\mathrm{a}}$ & $3.46 \pm 1.71$ \\
\hline$\alpha$-SMA-positive membrane area $(\%)$ & $6.33 \pm 0.86^{\mathrm{a}}$ & $4.33 \pm 1.51$ & $7.05 \pm 1.32^{\mathrm{a}}$ & $4.89 \pm 1.13$ \\
\hline
\end{tabular}

Values presented are the mean \pm standard deviation ( $\mathrm{n}=10$ per group). ${ }^{\mathrm{a}} \mathrm{P}<0.05$ compared with the sham surgery group at the same time-point.

those in the sham surgery group in the same time-point $(\mathrm{P}<0.05$; Table I).

Observation of histomorphology. On day 7 after arteriovenous fistula surgery in the experimental group, active smooth muscle cell proliferation was visible in the tunica membrane at the venous end, with significant intimal thickening, polypoid hyperplasia protruding into the cavity, and large amounts of smooth muscle cells and collagen fibers deposited in the hyperplastic tissue. On day 28 , the above pathological changes were more evident, with the lumen being more stenotic, but still able to allow the flow of blood. With double collagen and elastin staining, it was observed that the internal elastic layer was discontinuous with polypoid hyperplasia, and greater amounts of collagen fibers were deposited in the vein near the anastomotic stoma in the experimental group on days 7 and 28. By contrast, in the sham surgery group, the internal elastic layer was observed to be normal and continuous. Image analysis showed that the tunica intima and tunica media thickness and the neointimal area of the experimental group was gradually increased in week 1 and week 4, compared with those in the sham surgery group; the difference was statistically significant $(\mathrm{P}<0.05$; Table II, Figs. 1-3).

Immunohistochemistry. In the tunica intima and tunica media, the $\alpha$-SMA-positive areas near the anastomotic stoma in the experimental group were increased in size compared with those in the sham surgery group at the same time-point $(\mathrm{P}<0.05)$; in the experimental group, $\alpha$-SMA positive areas near the anastomotic stoma increased with time $(\mathrm{P}=0.52)$.

\section{Discussion}

An AVF remains the consensus-recommended vascular access for individuals requiring hemodialysis (15). It can cause the dilatation of native veins (16). Provision of a reliable and durable vascular access for hemodialysis continues to be a challenge for clinicians, with a retrospective review showing an overall primary failure rate of $5.5 \%$ (17). Approach through the radial artery distal is an effective and safe method for the salvage of nonmaturing radiocephalic arteriovenous fistulas (18). Inflow stenosis is frequently associated with fistula dysfunction (19). An animal model of autogenous fistula can simulate the dynamic changes in the local hemodynamics of an internal fistula and venous arterialization pathology, and facilitate the observation of the effects of intervention measures. Large and small animal models have advantages and disadvantages. In dogs, perianastomotic pannus has been found to be primarily composed of intimal smooth muscle cells, and neointimal thickening was significantly reduced in prosthetic arteriovenous fistulae (20). Various small animal models have been reported in the non-Chinese literature $(8,21-23)$. However, this type of modeling is complex and the success rate is low, and has not been reported previously in China. In the present study, a simplified model of autologous vascular fistula in rats with chronic renal failure was created, laying a foundation for further study of failure mechanisms and interventions.

An anastomosis method is often used in the establishment of the models, but the experimental results can be affected by the technology proficiency level of the practitioner. Application 


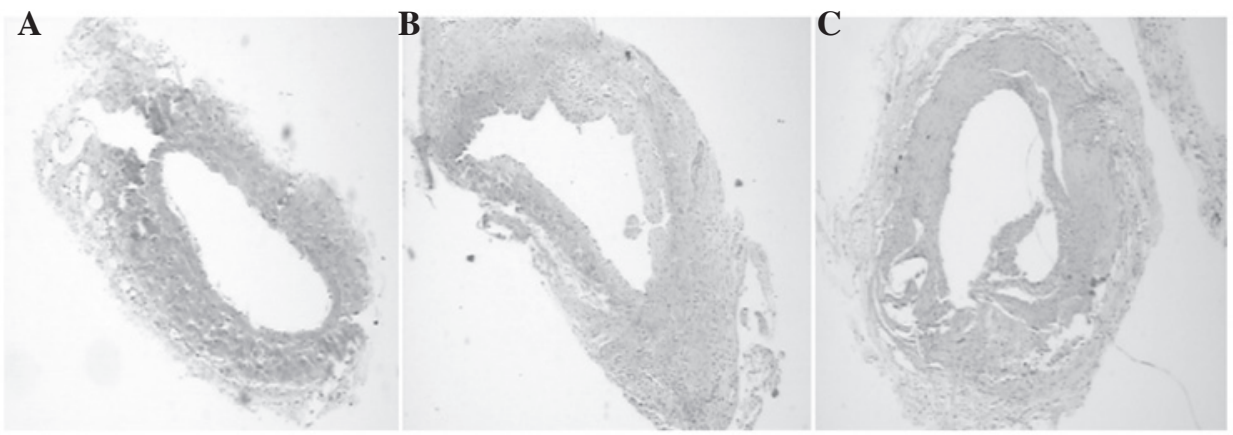

Figure 1. Intima stained with hematoxylin and eosin. Staining in (A) a vein from the sham group and veins near the anastomotic stoma in the experimental group at (B) 1 week after surgery and (C) 4 weeks after surgery (magnification, $\mathrm{x} 40$ ).

A

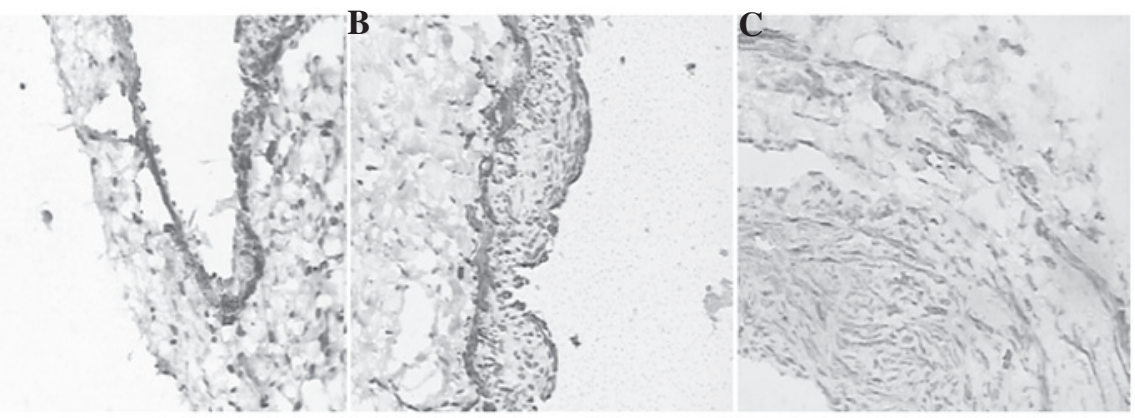

Figure 2. $\alpha$-SMA positive area of the vein tunica intima and tunica media membranes. Immunohistochemical staining of $\alpha$-SMA in (A) a vein in the sham group and veins near the anastomotic stoma in the experimental group at (B) 1 week after surgery and (C) 4 weeks after surgery (magnification, x200).
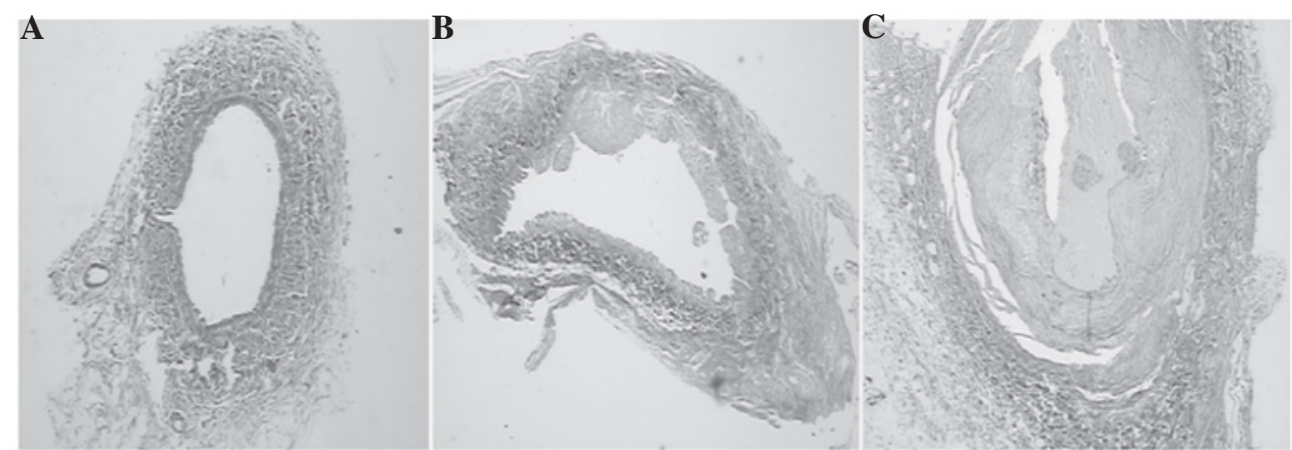

Figure 3. Intimal elastic and collagen fiber double staining in (A) a vein of the sham group and in veins near the anastomotic stoma of the experimental group at (B) 1 week after surgery and (C) 4 weeks after surgery (magnification, $\mathrm{x} 40$ ).

of a cuff technique in the anastomosis of blood vessels has been introduced in organ transplantation animal models $(3,24)$. The present study used a cuff technique to establish an AVF model in rats subjected to 5/6 nephrectomy, and may be considered to improve on previous animal models. The model has the following characteristics: i) routine modeling requires three surgical procedures and causes great trauma to the animal, while the current method causes minimal trauma, involves an simple dissection technique, with the anastomotic blood vessel being similar to that in the clinic, and is easy for the rats to endure; ii) anastomosis is conducted using a cuff technique without microsurgical suture, and has a short surgery time, a consistent anastomotic diameter and a high success rate (there were no failed cases in the experimental group); iii) the experimental results are less affected by the surgical technique and proficiency, with a low anastomotic blood leakage occurrence rate, high success rate of vein grafting and high fistula patency rate; however, attention must be paid when inserting the artery into the vein bridge, which requires two ligations, to avoid vein stump aneurysm and the creation of turbulence; iv) the disposable venous indwelling catheter used in the experimental group was a common clinical catheter, the specification of which may be selected according to the vascular caliber. It contacts only the carotid artery adventitia, that is, the carotid artery and external jugular vein anastomosis is connected between the intima, which does not readily cause thrombosis.

The 5/6 nephrectomy rat model was established successfully in this study. The blood urea nitrogen and creatinine levels of the experimental group had increased on day 7 after $5 / 6$ nephrectomy compared with those in the sham surgery 
group. On day 28 after the surgery, the blood urea nitrogen and creatinine values decreased slightly in the experimental group, but remained significantly higher than those in the sham surgery group. This indicates that acute decompensation may gradually transition to residual renal compensation, indicating an improved simulation of chronic renal insufficiency 28 days after surgery.

It is currently considered that the important pathological changes of intimal hyperplasia are the increase of vascular smooth muscle cells and extracellular matrix (13,25-28). The present study showed that there was intimal hyperplasia of the fistula in the first week after surgery, and the pathological features included smooth muscle cell proliferation, the accumulation of collagen fibers, extensive polypoidal hyperplasia, and evident stenosis in the fourth week after surgery. Staining showed that the elastic layer was not continuous following fistula surgery, suggesting that vessel fracture promotes smooth muscle cell migration to the intima. Immunohistochemistry showed that expression of $\alpha$-SMA in the experimental group gradually increased with time. The $\alpha$-SMA-positive area of the tunica intima and tunica media in the vein near the anastomotic stoma of the experimental group increased significantly compared with that in the sham operation surgery group at the same time-point in weeks 1 and 4 , indicating that the expression of $\alpha$-SMA may increase with increased intimal hyperplasia, consistent with previous studies (7,29-31).

In summary, a rat model of autologous arteriovenous fistula with chronic renal failure was successfully established by a cuff technique. This was indicated by the pathological changes to the vein, which were smooth muscle cell proliferation, evident collagen fiber deposition, and the expression of $\alpha$-SMA, by the appearance of the vessel and renal function. This model appears to provide a very good simulation of the pathological processes of chronic renal insufficiency with fistula. The establishment of the model is simple and easy to master, and can eliminate the effect of different anastomotic techniques on experimental research. Thus, it provides an ideal experimental animal model of early and metaphase chronic renal insufficiency for research into intimal hyperplasia, and is worthy of promotion.

\section{Acknowledgements}

This work is supported by the Fund of Science and Technology Bureau of Wenzhou City (grant no. Y20110129).

\section{References}

1. Gowda A, Pavan M and Babu K: Vascular access profile in maintenance hemodialysis patients. Iran J Kidney Dis 8: 218-224, 2014

2. Han M, Kim JD, Bae JI, Lee JH, Oh CK, Ahn C and Won JH: Endovascular treatment for immature autogenous arteriovenous fistula. Clin Radiol 68: e309-e315, 2013.

3. Kokubo T, Ishikawa N, Uchida H, et al: CKD accelerates development of neointimal hyperplasia in arteriovenous fistulas. J Am Soc Nephrol 20: 1236-1245, 2009.

4. Kakkos SK, Haddad GK, Stephanou A, Haddad JA and Shepard AS Routine preoperative venous and arterial mapping increases both, construction and maturation rate of upper arm autogenous arteriovenous fistulae. Vasc Endovascular Surg 45: 135-141, 2011.

5. Usta E, Elkrinawi R, Salehi-Gilani S, et al: Risk factors predicting the successful function and use of autogenous arteriovenous fistulae for hemodialysis. Thorac Cardiovasc Surg 61: 438-444, 2013
6. Chang CJ, Chen CC, Hsu LA, et al: Degradation of the internal elastic laminae in vein grafts of rats with aortocaval fistulae: potential impact on graft vasculopathy. Am J Pathol 174: 1837-1846, 2009

7. Chan CY, Chen YS, Ma MC and Chen CF: Remodeling of experimental arteriovenous fistula with increased matrix metalloproteinase expression in rats. J Vasc Surg 45: 804-811, 2007.

8. Castier Y, Lehoux S, Hu Y, Foteinos G, Tedgui A and Xu Q: Characterization of neointima lesions associated with arteriovenous fistulas in a mouse model. Kidney Int 70: 315-320, 2006.

9. Caplice NM, Wang S, Tracz M, Croatt AJ, Grande JP, Katusic ZS and Nath KA: Neoangiogenesis and the presence of progenitor cells in the venous limb of an arteriovenous fistula in the rat. Am J Physiol Renal Physiol 293: F470-F475, 2007.

10. Liang A, Wang Y, Han G, Truong L and Cheng J: Chronic kidney disease accelerates endothelial barrier dysfunction in a mouse model of an arteriovenous fistula. Am J Physiol Renal Physiol 304: F1413-1420, 2013.

11. Qin F, Dardik H, Pangilinan A, Robinson J, Chuy J and Wengerter K: Remodeling and suppression of intimal hyperplasia of vascular grafts with a distal arteriovenous fistula in a rat model. J Vasc Surg 34: 701-706, 2001.

12. National Research Council (US) Committee for the Update of the Guide for the Care and Use of Laboratory Animals: Guide for the Care and Use of Laboratory Animals. 8th edition. National Academies Press, Washington, 2011.

13. Hoch H, Stegbauer J, Potthoff SA, Hein L, Quack I, Rump LC and Vonend O: Regulation of renal sympathetic neurotransmission by renal $\alpha(2 \mathrm{~A})$-adrenoceptors is impaired in chronic renal failure. Br J Pharmacol 163: 438-446, 2011.

14. Sivanesan S, How TV, Black RA and Bakran A: Flow patterns in the radiocephalic arteriovenous fistula: an in vitro study. J Biomech 32: 915-925, 1999.

15. Jennings WC and Taubman KE: Alternative autogenous arteriovenous hemodialysis access options. Semin Vasc Surg 24: 72-81, 2011.

16. Georgakarakos EI, Kapoulas KC, Georgiadis GS, Tsangaris AS, Nikolopoulos ES and Lazarides MK: An overview of the hemodynamic aspects of the blood flow in the venous outflow tract of the arteriovenous fistula. J Vasc Access 13: 271-278, 2012.

17. Al-Benna S, Deardon D, Hamilton D and El-Enin H: Long-term outcome of upper limb autogenous arteriovenous fistulas for hemodialysis access. Saudi J Kidney Dis Transpl 24: 109-114, 2013.

18. Hsieh MY, Lin L, Tsai KC and Wu CC: Radial artery approach to salvage nonmaturing radiocephalic arteriovenous fistulas. Cardiovasc Intervent Radiol 36: 957-963, 2013.

19. Caeiro F, Carvalho D, Cruz J, Ribeiro Santos J and Nolasco F: Efficacy of percutaneous transluminal angioplasty on dysfunctional fistulae because of inflow stenosis. J Vasc Access 14: 231-238, 2013.

20. Gentile AT, Mills JL, Gooden MA, et al: Vein patching reduces neointimal thickening associated with prosthetic graft implantation. Am J Surg 176: 601-607, 1998.

21. Paszkowiak JJ and Dardik A: Arterial wall shear stress: observations from the bench to the bedside. Vasc Endovascular Surg 37: 47-57, 2003.

22. Corpataux JM, Haesler E, Silacci P, Ris HB and Hayoz D: Low-pressure environment and remodelling of the forearm vein in Brescia-Cimino haemodialysis access. Nephrol Dial Transplant 17: 1057-1062, 2002.

23. Guzman RJ, Krystkowiak A and Zarins CK: Early and sustained medial cell activation after aortocaval fistula creation in mice. J Surg Res 108: 112-121, 2002.

24. Mizuta T, Nakahara K, Shirakura R, Fujii Y, Kawaguchi A, Minami $\mathrm{M}$ and Kawashima Y: Total nomicrosuture technique for rat lung transplantation. J Thorac Cardiovase Surg 102: 159-160, 1991.

25. Roy-Chaudhury P, Sukhatme VP and Cheung AK: Hemodialysis vascular access dysfunction: a cellular and molecular viewpoint. J Am Soc Nephrol 17: 1112-1127, 2006.

26. Kelly B, Melhem M, Desai P, et al: Perivascular paclitaxel polymers reduce neointimal hyperplasia and venous stenosis in PTFE arteriovenous grafts: a possible solution for hemodialysis vascular access dysfunction. J Am Soc Nephrol 15: 25A, 2004.

27. Kuji T, Masaki T, Goteti K, et al: Efficacy of local dipyridamole therapy in a porcine model of arteriovenous graft stenosis. Kidney Int 69: 2179-2185, 2006.

28. Janardhanan R, Yang B, Vohra P, et al: Simvastatin reduces venous stenosis formation in a murine hemodialysis vascular access model. Kidney Int 84: 338-352, 2013. 
29. Muto A, Fitzgerald TN, Pimiento JM, et al: Smooth muscle cell signal transduction: implications of vascular biology for vascular surgeons. J Vasc Surg 45 (Suppl A): 15-24, 2007.

30. Roy-Chaudhury P, Miller M, Reaves A, et al: Adventitial fibroblasts contribute to venous neointimal hyperplasia in PTFE dialysis grafts. J Am Soc Nephrol 12: 301A, 2001.
31. Motwani JG and Topol EJ: Aortocoronary saphenous vein graft disease: pathogenesis, predisposition and prevention. Circulation 97: 916-931, 1998. 